\section{DIFERENÇAS NAS CARACTERÍSTICAS CLÍNICAS E EPIDEMIOLÓGICAS ENTRE OS CASOS NOTIFICADOS POR TUBERCULOSE NA ATENÇÃO PRIMÁRIA E TERCIÁRIA}

Differences in clinical and epidemiological features between
tuberculosis cases notified in primary and tertiary health care

Diferencias de las caracteristicas clinicas y epidemiológicas de los casos notificados de tuberculosis en la atención primaria y terciaria

\section{RESUMO}

Objetivo: Comparar, quanto aos aspectos clínicos, radiológicos e epidemiológicos, todos os casos de tuberculose (TB) diagnosticados nos serviços terciários de atenção à saúde com aqueles diagnosticados no nível primário de atenção à saúde do município de Vitória-ES. Métodos: Estudo de corte transversal, retrospectivo, com análises do banco de dados do SINAN, para identificar todos os casos residentes no município de Vitória-ES e diagnosticados com tuberculose nos anos de 2006 e 2007. Os pacientes foram divididos em dois grupos: os diagnosticados na Atenção Primária à Saúde (APS) e os diagnosticados na Atenção Terciária à Saúde (ATS). Para análise estatística, utilizou-se o programa Stata Corp 9.0. Resultados: Identificou-se um total de 338 pacientes; destes, 207 (61,24\%) diagnosticados na APS e $131(38,76 \%)$ na ATS. Observou-se um predomínio da forma clínica pulmonar em ambos os grupos, todavia, a extrapulmonar apresentou maior frequência no grupo da ATS. Quando analisados os resultados da baciloscopia de escarro, a positividade foi encontrada em 114 $(55,07 \%)$ dos pacientes da APS e em 57 (43,51\%) da ATS. Com relação à positividade da cultura, 119 (57,48\%) pacientes diagnosticados na APS e apenas 38 (29\%) na ATS tiveram resultado positivo. Conclusão: Há evidência de elevada proporção de pacientes com TB notificados como "casos novos" na Atenção Terciária no município e foi notado um predomínio da forma clínica pulmonar em ambos os grupos - APS e ATS. Todavia, a extrapulmonar apresentou maior frequência neste.

Descritores: Tuberculose; Atenção Primária à Saúde; Atenção Terciária à Saúde.

\section{ABSTRACT}

Objective: To compare, on the basis of clinical, radiological and epidemiological aspects, all cases of tuberculosis (TB) diagnosed in the tertiary healthcare services to those diagnosed at the primary healthcare providers in the municipality of Vitória-ES. Methods: Crosssectional, retrospective study, with analysis of the SINAN's database, to identify all cases among residents of the municipality of Vitória-ES and diagnosed with TB in 2006 and 2007. Patients were divided into two groups: cases diagnosed in Primary Health Care (PHC) and those diagnosed in Tertiary Health Care (THC). For statistical analysis, it was used the software STATA CORP 9.0. Results: 338 patients were identified; 207 (61.24\%) of these were diagnosed in PHC and 131 (38.76\%) in THC. The pulmonary form was predominant in both groups. However, the extrapulmonary form presented higher frequency in the THC group. When analyzing the results of sputum smear, the positivity was found in $114(55.07 \%)$ patients of the PHC and in 57 (43.51\%) of THC. Regarding the positive cultures, 119 (57.48\%) patients diagnosed in PHC and only 38 (29\%) in THC tested positive. Conclusion: There is evidence of high proportion of patients with TB communicated as "new cases" in THC in the city and it was observed a predominance of the pulmonary form in both groupsPHC and THC, although the extrapulmonary form was more frequent in the latter.

Descriptors: Tuberculosis; Primary Health Care; Tertiary Health Care.
Artigo Original
1) Universidade Federal do Espírito Santo UFES - Vitória-ES - Brasil
Recebido em: 06/07/2012 Revisado em: 22/11/2012 Aceito em: 02/01/2013 


\section{RESUMEN}

Objetivo: Comparar todos los casos de tuberculosis (TB) diagnosticados en los servicios terciarios de salud con aquellos diagnosticados en nivel primario de salud del municipio de Vitoria-ES acerca de los aspectos clínicos, radiológicos y epidemiológicos Métodos: Estudio trasversal, retrospectivo, con análisis del banco de datos del SINAN para identificar todos los casos residentes en el municipio de Vitoria-ES y diagnosticados con tuberculosis en los años 206 y 2007. Los pacientes fueron divididos en dos grupos: los diagnosticados en la Atención Primaria en Salud (APS) y los diagnosticados en la Atención Terciaria en Salud (ATS). Para el análisis estadístico se utilizó el programa Stata Corp 9.0. Resultados: Se identificó un total de 338 pacientes; de esos, 207 (61,24\%) fueron diagnosticados en la APS y 131 (38,76\%) en la ATS. Se observó un predominio de la forma clínica pulmonar en ambos grupos, sin embargo, la extra pulmonar presentó mayor frecuencia en el grupo de la ATS. Cuando analizados los resultados de la baciloscopia de esputo, la positividad fue encontrada en 114 (55,07\%) de los pacientes de la APS y en 57 (43,51\%) de la ATS. Respecto la positividad de la cultura, 119 (57,48\%) pacientes diagnosticados en la APS y apenas 38 (29\%) en la ATS tuvieron resultado positivo. Conclusión: Hay evidencia de elevada proporción de pacientes con TB notificados como "casos nuevos" en la Atención Terciaria del municipio y fue observado un predominio de la forma clínica pulmonar en ambos grupos - APS y ATS. Sin embargo, la extra pulmonar presentó mayor frecuencia en este.

Descriptores: Tuberculosis; Atención Primaria de Salud; Atención Terciaria de Salud

\section{INTRODUÇÃO}

A tuberculose (TB) é uma das mais antigas doenças infecciosas do mundo. É potencialmente curável graças ao seu tratamento medicamentoso eficaz, porém, permanece como um grave problema de saúde pública. Ela é causada pelo Mycobacterium tuberculosis e, de acordo com estimativas da Organização Mundial de Saúde (OMS), 1/3 da população mundial está infectada pelo bacilo. Apesar da redução progressiva nas taxas de incidência nos últimos 10 anos, o Brasil continua sendo um dos 22 países responsáveis por $80 \%$ dos casos de tuberculose em todo o mundo. Em 2009, a OMS estimou uma taxa de incidência de 45 casos por 100.000 habitantes no Brasil e, de acordo com dados do Ministério da Saúde, essa taxa foi de 37 casos por 100.000 habitantes no mesmo ano ${ }^{(1-3)}$.

No estado do Espírito Santo, em 2010, foram notificados 1.489 casos novos de TB de todas as formas clínicas (taxa de incidência de 35,3/100.000 habitantes). Dos cinco municípios que compõem a Região Metropolitana da Grande Vitória, o município de Vitória, a capital do estado, é o menor deles, com uma população de 320.000 habitantes. Em 2010, foram registrados 158 casos novos da doença nesse município, produzindo uma taxa de incidência de 49,3/100.000 habitantes ${ }^{(3)}$.

Diante desse quadro de emergência sanitária declarado pela OMS, o Ministério da Saúde lançou o Programa Nacional de Controle da Tuberculose (PNCT), em 1999, e definiu a doença como prioridade entre as políticas governamentais de saúde, estabelecendo diretrizes para as ações e fixando metas para o alcance de seus objetivos ${ }^{(4)}$. As metas internacionais, estabelecidas pela OMS e pactuadas pelo governo brasileiro, são: diagnosticar $70 \%$ dos casos estimados e curá-los em $85 \%{ }^{(2)}$.

Em 2004, o PNCT reconheceu a importância de se descentralizar as ações de combate à TB para o nível de assistência da Atenção Primária à Saúde (APS), ressaltando a contribuição do Programa de Agentes Comunitários de Saúde (PACS) e da Estratégia de Saúde da Família (ESF) na ampliação do acesso ao diagnóstico e ao tratamento ${ }^{(5)}$.

Porém, muitas vezes, alguns casos deixam de ser precocemente diagnosticados e tratados na APS, e passam a ser diagnosticados com atraso nos serviços de urgência e emergência. Esse quadro, além de definir uma grave deficiência no sistema de saúde em relação à atenção primária, traz consequências que, muitas vezes, põem em risco a vida do paciente, uma vez que o atraso no diagnóstico e no início do tratamento aumenta a gravidade da morbimortalidade por $\mathrm{TB}$, bem como o risco de transmissão na comunidade ${ }^{(6,7)}$. Dessa forma, ocorre uma incapacidade de se controlar essa endemia, possibilitando a perpetuação da cadeia de transmissão(7).

No município de Vitória-ES, a rede de Atenção Terciária à Saúde (ATS) é uma importante porta de entrada para os pacientes, representando um percentual em torno de $30 \%$ dos casos novos notificados. Assim, o objetivo do presente estudo é comparar, quanto aos aspectos clínicos e epidemiológicos, todos os casos de TB diagnosticados nos serviços terciários da atenção a saúde com aqueles diagnosticados no nível primário de atenção à saúde do município.

\section{MÉTODOS}

Delineou-se um estudo de corte transversal, retrospectivo, com análises de dados secundários obtidos do banco de dados do Sistema de Informação de Agravos de Notificação (SINAN) do Espírito Santo para identificar todos os casos de TB notificados no município de VitóriaES nos anos de 2006 e 2007.

Utilizou-se o campo "unidade de notificação" para a identificação e formação de dois grupos: o grupo cujo 
diagnóstico havia sido realizado na APS e o grupo cujo diagnóstico foi realizado nos hospitais que compõem a rede terciária do município de Vitória-ES: Hospital Universitário Cassiano Antônio de Moraes (HUCAM), Hospital Infantil Nossa Senhora da Glória, Santa Casa de Misericórdia de Vitória, Hospital Santa Rita de Cássia e Hospital da Polícia Militar.

Como critério de inclusão, estabeleceu-se que os pacientes deveriam ter sido notificados como caso novo da doença, ser maiores de 18 anos e residir em Vitória-ES. Caso tivesse sido notificado primeiramente em um nível de atenção e, em seguida, em outro, ele era excluído do estudo. Com isso, ressalta-se que todos os casos da ATS foram notificados somente no hospital, não havendo transferência da APS para a ATS.

Para comparação quanto aos aspectos clínicos e epidemiológicos, estudaram-se as seguintes variáveis: idade, sexo, escolaridade, resultado da sorologia para o vírus HIV, radiografia de tórax e forma clínica da doença. Os dados laboratoriais (baciloscopia e cultura) foram complementados pelos registros obtidos diretamente no Laboratório de Microbiologia do Núcleo de Doenças Infecciosas da Universidade Federal do Espírito Santo (UFES).

Todas as informações obtidas deram origem a um banco de dados armazenado no software Microsoft Excel e, posteriormente, transferido para o programa estatístico
Stata Corp 9.0, para o cálculo das frequências absoluta e relativa. Além disso, calculou-se a proporção de doentes classificados na APS e na ATS, através da razão de prevalência, com intervalos de confiança de $95 \%$ da distribuição binomial.

Todos os procedimentos éticos foram rigorosamente cumpridos, como a aprovação do projeto pelo Comitê de Ética em Pesquisa em Seres Humanos do Centro de Ciências da Saúde da UFES, com número de registro 064/08.

\section{RESULTADOS}

Foi estudado um total de 338 pacientes; destes, 207 $(61,2 \%)$ receberam diagnóstico na APS e $131(38,8 \%)$ na ATS do município de Vitória-ES. Houve predominância do sexo masculino nos dois grupos pesquisados. Na APS, $140(67,6 \%)$ pacientes eram homens e na ATS, $81(61,8 \%)$. Com referência à escolaridade, nos dois grupos, houve a prevalência de pacientes com Ensino Fundamental e Médio. No teste de tendência, o valor de $\mathrm{z}$ indicou a rejeição de hipótese nula, ou seja, o teste rejeitou a hipótese de não tendência entre as categorias da variável escolaridade (Tabela I). A média de idade nos dois grupos foi de 38 anos, havendo predominância de casos na faixa etária entre 16 e 59 anos de idade. Dos pacientes diagnosticados na APS, $113(54,6 \%)$ pertenciam à faixa etária menor de 38 anos, enquanto, entre os diagnosticados na ATS, $74(56,5 \%)$ estavam na faixa etária maior ou igual a 38 anos de idade.

Tabela I - Aspectos sociodemográficos dos casos de TB notificados nas APS e ATS, no município de Vitória-ES, entre os anos de 2006 e 2007.

\begin{tabular}{lccccc}
\hline & APS & $\mathbf{\%}$ & ATS & $\mathbf{\%}$ & RP (IC 95\%) \\
\hline Sexo & & & & & \\
$\quad$ Feminino & 67 & 32,36 & 50 & 38,16 & $0.77(0.47-1.25) *$ \\
$\quad$ Masculino & 140 & 67,64 & 81 & 61,84 & \\
& & & & & $* *$ \\
Escolaridade & 7 & 3,38 & 5 & 3,81 & \\
$\quad$ Nenhuma & 109 & 52,65 & 52 & 39,69 & \\
Ensino Fundamental & 21 & 10,14 & 14 & 10,68 & \\
Ensino Médio & 9 & 4,34 & 7 & 3,34 & \\
Ensino Superior & 10 & 4,83 & 4 & 37,05 & \\
Não se aplica & 51 & 24,66 & 49 & & \\
Não informado & & & & & \\
Idade & 113 & 54,6 & 57 & 43,5 & \\
$\quad<38$ anos & 94 & 45,4 & 74 & 56,5 & \\
$\geq 38$ anos & & 100 & 131 & 100 & \\
\hline
\end{tabular}

$* \mathrm{p}$ valor $=0,27 \quad * *_{\mathrm{z}}=0,01$ 
Na Tabela II, pode-se observar que 53,5\% (181) dos pacientes realizaram a sorologia para HIV. Desses, 24,31\% (44) tiveram o resultado do teste positivo. No grupo da APS, 51,21\% (106) realizaram o teste e 6,6\% (7/207) do total desse grupo tiveram resultado positivo. Na ATS, $57,2 \%$ (75) realizaram o teste, com 28,25\% (37/131) de positividade no grupo. Esses percentuais não se referem à taxa de coinfecção TB/HIV, uma vez que 48,79\% (101) na APS e $42,75 \%$ (56) na ATS não realizaram a sorologia.
Ao se verificarem os resultados dos exames de raios-X, observa-se que 96,13\% (199) dos pacientes da APS possuíam resultados suspeitos de TB e 2,38\% (6) apresentavam resultados normais. Na ATS, $91(69,46 \%)$ pacientes também possuíam exame de raios-X suspeito e 15 $(11,46 \%)$ apresentavam resultados normais.

Com relação à forma clínica da doença, os dois grupos apresentaram predomínio da forma clínica pulmonar. No entanto, os pacientes diagnosticados na ATS apresentaram

Tabela II - Aspectos clínicos dos casos notificados de TB nas APS e ATS, no município de Vitória-ES, entre os anos de 2006 e 2007.

\begin{tabular}{lccccc}
\hline & APS & $\mathbf{\%}$ & ATS & $\mathbf{\%}$ & RP (IC 95\%) \\
\hline Sorologia para HIV & & & & & \\
Positivo & 7 & 3,39 & 37 & 28,25 & $0.088(0.032-0.21)^{*}$ \\
Negativo & 99 & 47,82 & 38 & 29 & \\
Não realizado & 101 & 48,79 & 56 & 42,75 & \\
& & & & & \\
Raio-X & & & & & \\
Suspeito & 199 & 96,13 & 91 & 69,46 & $10.93(4.76-27.94) *$ \\
Normal & 6 & 2,89 & 15 & 11,46 & \\
Outra patologia & 0 & 0 & 5 & 3,82 & \\
Não realizado & 2 & 0,98 & 20 & 15,26 & \\
& & & & & \\
Forma Clínica & 173 & 83,57 & 61 & 46,56 & $0,17(0,1-0,29) *$ \\
Pulmonar & 25 & 12,07 & 49 & 37,4 & \\
Extrapulmonar & 9 & 4,36 & 21 & 16,04 & \\
Pulmonar + Extrapulmonar & & & & \\
& 207 & 100 & 131 & 100 & \\
\hline
\end{tabular}

*p-valor $=0,00$

Tabela III - Características laboratoriais dos casos notificados de TB nas APS e ATS, no município de Vitória-ES, entre os anos de 2006 e 2007.

\begin{tabular}{lccccc}
\hline & APS & $\mathbf{\%}$ & ATS & $\mathbf{\%}$ & RP(IC 95\%) \\
\hline Baciloscopia & & & & & \\
$1+$ & 17 & 8,21 & 8 & 6,1 & $0,16(0,09-0,28)^{*}$ \\
$2+$ & 30 & 14,5 & 5 & 3,81 & \\
$3+$ & 67 & 32,36 & 9 & 6,87 & \\
Negativa & 77 & 37,2 & 74 & 56,49 & \\
Não informado & 16 & 7,73 & 35 & 26,73 & \\
Cultura & & & & \\
Positiva & 119 & 57,48 & 38 & 29 & $0,3(0,18-0,49)^{*}$ \\
Negativa & 72 & 34,78 & 58 & 44,27 & \\
Não informado & 16 & 7,74 & 35 & 26,73 & \\
& & & & & \\
\hline
\end{tabular}

*p-valor $=0,00$ 
a forma extrapulmonar com maior frequência - 37,4\% (49)

- sendo a diferença estatisticamente significativa $(p=0,00)$.

Quando analisados os resultados da baciloscopia de escarro, observa-se que, dos pacientes diagnosticados na APS, 77 (37,2\%) possuíam baciloscopia negativa e 114 $(55,07 \%)$ apresentavam baciloscopia positiva; destes, 67 $(32,36 \%)$ possuíam baciloscopia positiva $3+$. Na ATS, $74(56,49 \%)$ possuíam resultado negativo deste exame e $9(6,87 \%)$ apresentavam resultado positivo. Quanto à cultura, 119 (57,48\%) pacientes diagnosticados na APS apresentavam cultura positiva, enquanto apenas $38(29 \%)$ dos diagnosticados na ATS possuíam esse mesmo resultado (Tabela III).

\section{DISCUSSÃO}

A detecção dos casos novos de TB depende fundamentalmente da qualificação e educação continuada das equipes de saúde, uma vez que a maioria dos casos não requer exames sofisticados; um exame de raios-X e um de escarro são suficientes para uma avaliação inicial do paciente $^{(8)}$. Quando a APS falha, seja pela dificuldade de acesso ao serviço de saúde ou pela falta de capacitação das equipes de saúde em identificar os sintomáticos respiratórios, um aumento no número de casos de TB diagnosticados nos hospitais é observado ${ }^{(9)}$. Um estudo realizado na cidade de Vitória-ES, em 2009, relacionado ao atraso no diagnóstico para tuberculose na atenção básica de saúde, verificou um atraso total de 110 dias $^{(10)}$.

No período de investigação do presente estudo, pôde-se observar que a maioria dos casos notificados foi pela APS, porém, notou-se uma parcela importante de casos diagnosticados na ATS. Isso reflete a dificuldade do sistema de saúde em identificar precocemente os casos de tuberculose, gerando um número expressivo de hospitalizações.

Sobre a caracterização dos casos, quanto ao sexo, o atual estudo demonstrou que houve maior predomínio de pacientes do sexo masculino diagnosticados com TB nos dois grupos, acompanhando uma tendência no Brasil e no mundo ${ }^{(1)}$. Quanto à escolaridade, os dois grupos se concentraram principalmente no nível de Ensino Fundamental, o que revela o baixo nível de escolaridade dos pacientes estudados, confirmando a tendência secular da doença, cuja incidência é maior em populações mais desfavorecidas ${ }^{(11)}$.

A distribuição dos pacientes nos dois grupos investigados na presente pesquisa, com relação à faixa etária, esteve entre 16 e 59 anos, estando de acordo com outros estudos $^{(11,12)}$ e se assemelhando com a realidade brasileira, em que a TB atinge principalmente a população economicamente ativa ${ }^{(11)}$, conferindo significado social à doença.

A OMS estima que, entre os 9,27 milhões de casos de TB incidentes em 2007, 1,37 milhões (14,8\%) eram HIV positivos em todo mundo. No Brasil, a incidência da doença em pacientes HIV positivos se assemelha às taxas mundiais, atingindo $14 \%$ dos casos de $\mathrm{TB}^{(1)}$. No presente estudo, apesar de se mostrar uma baixa testagem para o HIV nos dois níveis de atenção, observou-se uma predominância dos pacientes HIV positivos na ATS e uma necessidade de se aumentar a oferta da sorologia na APS. Em um estudo realizado na cidade de São Paulo-SP, a sorologia para HIV obteve resultado positivo em $42 \%$ dos pacientes internados em um hospital universitário ${ }^{(9)}$. Esse fato demonstra a maior gravidade no quadro clínico desses pacientes, devido ao seu estado de imunossuprimido, sendo necessária sua internação.

$\mathrm{O}$ atual estudo também mostrou um número grande de sorologias para HIV não realizadas, o que é muito preocupante, pois, segundo o Manual Técnico para o Controle da Tuberculose, a todo doente com diagnóstico de tuberculose confirmado deve ser oferecido o teste sorológico anti-HIV $^{(8)}$. Em pessoas diagnosticadas com o teste HIV positivo, deve-se avaliá-las para afastar a suspeição de $\mathrm{TB}^{(8)}$, uma vez que essa doença é a primeira causa de mortalidade entre pacientes com AIDS. Esse resultado aponta uma falha do serviço, que se organiza em ações programáticas, em vez de ofertar um cuidado integral ao paciente que acessa o serviço de saúde.

Diante disso, percebe-se, ainda, a falta de articulação entre o programa de tuberculose e o programa de HIV/ AIDS. Em locais onde esses programas funcionam coordenadamente, os pacientes com HIV são periodicamente avaliados, o que minimiza e acelera o tratamento da tuberculose, evitando que tenham seu estado de saúde agravado e venham a necessitar de internação. Estudos apontam para os benefícios da maior integração entre os dois programas ${ }^{(13-17)}$.

Apesar de a forma clinica pulmonar ter sido predominante nos dois níveis de atenção na atual pesquisa, observou-se a normalidade maior desse exame na ATS, mostrando que os casos de maior complexidade ficam concentrados nesse nível de atenção, provavelmente pela maior resolutividade e experiência desses serviços.

Em conjunto com a história clínica do paciente, a baciloscopia direta do escarro e a cultura são métodos fundamentais para o diagnóstico da TB, porque permitem descobrir as fontes mais importantes de infecção, os casos bacilíferoscavitários ${ }^{(18)}$. O presente estudo observou que mais da metade dos pacientes diagnosticados nas Unidades Básicas de Saúde apresentavam cultura e baciloscopia 
positiva, e a maioria possuía baciloscopia $3+$. Um estudo realizado na cidade de Vitória-ES relacionou a contagem de bacilos da tuberculose presentes na baciloscopia com a doença cavitária e demonstrou que o número de bacilos é muito maior em pacientes com lesões cavitárias do que em pacientes não cavitários ${ }^{(19)}$

Dessa forma, vê-se que muitos pacientes pertencentes ao grupo da APS já apresentavam estado avançado da doença, comprovando, mais uma vez, o atraso no diagnóstico desses pacientes. O fato de a maioria dos pacientes da ATS terem apresentado a cultura e a baciloscopia de escarro negativas pode ser devido ao número importante de formas extrapulmonares ou ao fato de terem sido referenciados a esses serviços, para melhor diagnóstico da doença; além dos pacientes HIV positivos presentes na ATS, que necessitam de melhor abordagem no diagnóstico.

Os achados da presente pesquisa são interessantes, pois o que se apontava como uma possível inversão na hierarquização do atendimento dos casos, com a prevalência observada de $38 \%$ de casos notificados na ATS, parece ser a dificuldade da APS em notificar os casos de TB extrapulmonar, haja vista que esses pacientes necessitam de exames mais complexos e são rotineiramente enviados para a ATS.

Um trabalho realizado em Ribeirão Preto-SP ${ }^{(20)}$, entre junho de 2006 e julho de 2007, objetivou identificar e analisar a porta de entrada no sistema de saúde para o diagnóstico da TB. Para tanto, realizou-se entrevista estruturada com 100 doentes diagnosticados no período do estudo. Destes, $66 \%$ procuraram por serviços de atenção primária e 34\%, por serviços de nível secundário e terciário. Apesar de terem procurado atendimento na atenção primária, o diagnóstico foi realizado principalmente em unidades de nível secundário e terciário, abrangendo $73 \%$ do total dos diagnósticos realizados. A atenção básica foi menos representativa, com um total de apenas $16 \%$ dos casos, comprovando a dificuldade da APS na realização do diagnóstico para TB.

\section{CONCLUSÃO}

O estudo evidenciou a elevada proporção de pacientes com TB notificados como "casos novos" na Atenção Terciária no município. Ao se comparar os casos quantos às características clínicas e epidemiológicas, notou-se um predomínio da forma clínica pulmonar em ambos os grupos. Todavia, a extrapulmonar apresentou maior frequência no grupo da ATS do que na APS.

Essa informação provavelmente refletiu nos dados microbiológicos da baciloscopia do escarro e da cultura, em que a positividade foi proporcionalmente maior na APS, assim como os achados suspeitos de TB da radiografia do tórax. Verificou-se, também, baixa investigação diagnóstica de infecção pelo HIV entre os pacientes com TB em ambos os grupos. Entretanto, entre os pacientes da ATS, o resultado foi positivo se comparado ao grupo da APS.

Observa-se a necessidade de investimento governamental na capacitação dos profissionais da APS para realizarem precocemente o diagnóstico da TB pulmonar e extrapulmonar na comunidade. Acredita-se que uma intensificação na realização da busca ativa de sintomáticos respiratórios na comunidade e uma investigação periódica entre pacientes infectados pelo HIV, para a detecção precoce e o acompanhamento dos casos, poderiam reduzir as internações para diagnóstico da TB e, consequentemente, diminuir as taxas de morbimortalidade pela TB em VitóriaES.

\section{REFERÊNCIAS}

1. World Health Organization. Global Tuberculosis Control. Geneva: WHO Report, 2010.

2. Conde MB, Melo FAF, Marques AMC, Cardoso NC, Pinheiro VGF, Dalcin PTR, et al . III Diretrizes para Tuberculose da Sociedade Brasileira de Pneumologia e Tisiologia. J Bras Pneumol. 2009;35(10):1018-48.

3. Ministério da Saúde (BR). Sistema de Informações sobre Mortalidade. e Ministério da Saúde. Série histórica de doenças de notificação compulsória por UF, 1980-2001. Brasília: Ministério da Saúde; 2012.

4. Silva JR JB. Tuberculose: Guia de Vigilância Epidemiológica. J Bras Pneumol. 2004;30(1):57-83.

5. Ministério da Saúde (BR). Pacto dos Indicadores da Atenção Básica. Informe da Atenção Básica. Brasília: Ministério da Saúde; 2008.

6. Kiwuwa MS, Charles K, Harriet MK. Patient and health service delay in pulmonary tuberculosis patients attending a referral hospital: a cross-sectional study. BMC Public Health. 2005;5(122):1-7.

7. Millen SJ, Uys PW, Hargrove J, Helden PDV, Williams BG. The Effect of Diagnostic Delays on the Drop-Out Rateand the Total Delay to Diagnosis of Tuberculosis. Plos One. 2008;3(4):1-10.

8. Ministério da Saúde (BR). Programa Nacional de Controle da Tuberculose. Manual de Recomendações para o Controle da Tuberculose no Brasil. Brasília; 2010 .

9. Ribeiro AS, Matsui TN. Admission for tuberculosis to a university hospital. J Bras Pneumol. 2003; 29(1): 9-14. 
10. Maciel EL, Golub JE, Peres RL, Hadad DJ, Fávero JL, Molino LP, Bae JW, Moreira CM, Detoni Vdo V, Vinhas SA, Palaci M, Dietze R. Delay in diagnosis of pulmonary tuberculosis at a primary health clinic in Vitoria, Brazil. Int $\mathrm{J}$ Tuberc Lung Dis. 2010;14(11):1403-10.

11. Severo NPF, Leite CQF, Capela MV, Simões MJS. Características clínico-demográficas de pacientes hospitalizados com tuberculose no Brasil, no período de 1994 a 2004. J Bras Pneumol. 2007;33(5):565-571.

12. Maciel ELN, Silva AP, Meireles W, Fiorotti K, Hadad DJ, Dietze R. Tratamento supervisionado em pacientes portadores de tuberculose utilizando supervisores domiciliares em Vitória, Brasil. J Bras Pneumol. 2008; 349 (7): 506-513.

13. Carvalho LG, Buani AZ, Zöllner MSAC, Scherma AP. Co-infecção por Mycobacterium tuberculosis e vírus da imunodeficiência humana: uma análise epidemiológica em Taubaté (SP). J Bras Pneumol. 2006; 32 (5): 424-9;

14. Muniz JN, Ruffino-Netto A, Villa TCS, Yamamura M, Arcencio R, Cardozo-Gonzales RI. Aspectos epidemiológicos da co-infecção tuberculose e vírus da imunodeficiência humana em Ribeirão Preto (SP), de 1998 a 2003. J Bras Pneumol. 2006;3 (6):529-34.

15. Coetzee D, Hilderbrand K, Goemaere E, Matthys F, Boelaer M. Integrating tuberculosis and HIV care in the primary care setting in South Africa. Tropical Medicine and International Health. 2004;9(6):a11-a15.

16. Harris JB, Hatwiinda SM, Randels KM, Chi BH, Kancheya NG, Jham MA, et. al. Early lessons from the integration of tuberculosis and HIV services in primary care centers in Lusaka, Zambia. Int J Tuberc.Lung. Dis. 2008;1(7): 773-9.

17. Perumal R, Padayatchi N, Stiefvater E. The whole is greater than the sum of the parts: Recognising missed opportunities for an optimal response to the rapidly maturing TB-HIV co-epidemic in South Africa. BMC Public Health. 2009;9:243.

18. Palaci M, Dietze R, Hadad DJ, Ribeiro FKC, Peres RL, Vinhas AS, et al. Cavitary Disease and Quantitative sputum bacillary load in cases of pulmonary tuberculosis. J Clin Microbiol. 2007;45(12):4064-6.

19. Conde MB, Melo FAF de, Marques AMC, Cardoso NC, Pinheiro VGF, Dalcin PTR et AL. III Consenso Brasileiro de Tuberculose: Diretrizes Brasileiras para Tuberculose. J Bras Pneumol. 2009;35(10):1018-48.

20. Oliveira MF, Arcêncio RA, Ruffino-Netto A, Scatena LM, Palha PF, Villa TCS. A porta de entrada para o diagnóstico da tuberculose no sistema de saúde de Ribeirão Preto/SP. Rev Esc Enferm USP. 2011; 45(4):898-904.

\section{Endereço primeiro autor:}

Pâmela Curbani

Laboratório de Epidemiologia / Programa de Pós-

Graduação em Saúde Coletiva / CCS -UFES

Av. Marechal Campos, 1468

Bairro: Maruípe

CEP: 29040-091 - Vitória-ES - Brasil

E-mail: pam.curbani@yahoo.com.br

\section{Endereço para correspondência:}

Ethel Leonor Noia Maciel.

Núcleo de Doenças Infecciosas / Centro de Ciências da Saúde / UFES

Av. Marechal Campos, 1468

Bairro: Maruípe

CEP: 29040-091 - Vitória-ES - Brasil

E-mail: ethel.maciel@gmail.com 